\title{
ASYMPTOTIC DISTRIBUTIONS OF THE ZEROS OF CERTAIN CLASSES OF HYPERGEOMETRIC FUNCTIONS AND POLYNOMIALS
}

\author{
H. M. SRIVASTAVA, JIAN-RONG ZHOU, AND ZHI-GANG WANG
}

\begin{abstract}
The main object of this paper is to consider the asymptotic distribution of the zeros of certain classes of the Clausenian hypergeometric ${ }_{3} F_{2}$ functions and polynomials. Some classical analytic methods and techniques are used here to analyze the behavior of the zeros of the Clausenian hypergeometric polynomials:
\end{abstract}

$$
{ }_{3} F_{2}(-n, \tau n+a, b ; \tau n+c,-n+d ; z),
$$

where $n$ is a nonnegative integer. Some families of the hypergeometric ${ }_{3} F_{2}$ functions, which are connected (by means of a hypergeometric reduction formula) with the Gauss hypergeometric polynomials of the form

$$
{ }_{2} F_{1}(-n, k n+l+1 ; k n+l+2 ; z),
$$

are also investigated. Numerical evidence and graphical illustrations of the clustering of zeros on certain curves are generated by Mathematica (Version 4.0).

\section{INTRODUCTION}

A generalized hypergeometric ${ }_{p} F_{q}$ function with $p$ numerator parameters $\alpha_{1}, \cdots, \alpha_{p}$ and $q$ denominator parameters $\beta_{1}, \cdots, \beta_{q}$ is defined by (see, for details, [1] and [17)

$$
\begin{gathered}
{ }_{p} F_{q}\left[\begin{array}{c}
\alpha_{1}, \cdots, \alpha_{p} ; \\
\beta_{1}, \cdots, \beta_{q} ;
\end{array}\right]=\sum_{k=0}^{\infty} \frac{\left(\alpha_{1}\right)_{k} \cdots\left(\alpha_{p}\right)_{k}}{\left(\beta_{1}\right)_{k} \cdots\left(\beta_{q}\right)_{k}} \frac{z^{k}}{k !} \\
\left(p, q \in \mathbb{N}_{0} ; p \leqq q+1 ; p \leqq q \text { and }|z|<\infty ; p=q+1 \text { and }|z|<1\right),
\end{gathered}
$$

provided that no zeros appear in the denominator. Here, and in what follows,

$$
\mathbb{N}_{0}:=\{0,1,2, \cdots\}=\mathbb{N} \cup\{0\} \quad(\mathbb{N}:=\{1,2,3, \cdots\})
$$

Received by the editor December 1, 2009 and, in revised form, January 7, 2010.

2010 Mathematics Subject Classification. Primary 33C05, 33C20; Secondary 30C15, 33C45.

Key words and phrases. Generalized hypergeometric functions, Clausenian hypergeometric function, Gauss hypergeometric polynomials, asymptotic distribution of zeros, zeros of ${ }_{3} F_{2}(-n, \tau n+a, b ; \tau n+c,-n+d ; z)$, zeros of ${ }_{3} F_{2}(-n, a, b ; c, d ; z)$, Jacobi polynomials, Rice polynomials, Pasternack polynomials, hypergeometric reduction formulas, Euler-Mascheroni constant, Vitali's theorem, Hurwitz's theorem, Eneström-Kakeya theorem, Mathematica (Version 4.0).

(C)2011 American Mathematical Society Reverts to public domain 28 years from publication 
and $(\lambda)_{\nu}$ denotes the Pochhammer symbol (or the shifted factorial, since $(1)_{n}=n$ ! for $n \in \mathbb{N}$ ) defined, in terms of the familiar Gamma functions, by

$$
(\lambda)_{\nu}:=\frac{\Gamma(\lambda+\nu)}{\Gamma(\lambda)}= \begin{cases}1 & (\nu=0 ; \lambda \in \mathbb{C} \backslash\{0\}) \\ \lambda(\lambda+1) \cdots(\lambda+n-1) & (\nu=n \in \mathbb{N} ; \lambda \in \mathbb{C}),\end{cases}
$$

$\mathbb{C}$ being the set of complex numbers.

If one of the numerator parameters $\alpha_{1}, \cdots, \alpha_{p}$ is equal to a negative integer or zero, say

$$
\alpha_{1}=-n \quad\left(n \in \mathbb{N}_{0}\right),
$$

then the series terminates and reduces to a polynomial of degree $n$ in $z$. The natural question that arises in connection with any polynomials is the correlative properties of its zeros (see, for example, [3, 9, 11, 21]).

The hypergeometric polynomials whose properties are best known and understood, including the location of their zeros and their asymptotic zero distribution, are those in the ${ }_{1} F_{1}$ (Kummer and Whittaker) and ${ }_{2} F_{1}$ (Gauss) classes (see, for details, 20]), mainly because of their connection with orthogonal polynomials 13. In particular, in the case of the Gauss hypergeometric ${ }_{2} F_{1}$ polynomials, their connection with the classical Jacobi polynomials $P_{n}^{(\alpha, \beta)}(z)$ given by (see [19, p. 62] and [18, p. 35])

$$
P_{n}^{(\alpha, \beta)}(z)=(-1)^{n} P_{n}^{(\beta, \alpha)}(-z)=\left(\begin{array}{c}
\alpha+n \\
n
\end{array}\right){ }_{2} F_{1}\left[\begin{array}{rr}
-n, \alpha+\beta+n+1 ; & 1-z \\
\alpha+1 ; & \frac{1-z}{2}
\end{array}\right]
$$

has led to a great deal of significant information about their zeros. For the ${ }_{3} F_{2}$ polynomials, however, there is no connection (in general) with the classical orthogonal polynomials, and the knowledge of the location of their zeros is restricted to some rather special polynomial classes (see, for example, [4, 5, 7, 8, 15]; see also [18, pp. 140 and 183] for the Clausenian hypergeometric ${ }_{3} F_{2}$ representations of the Rice polynomials and the Pasternack polynomials, respectively).

In the present paper, we propose to derive the asymptotic results for the zeros of the following general family of hypergeometric ${ }_{3} F_{2}$ polynomials (see also Section 2 and [6] for some of the various known or new special cases):

$$
{ }_{3} F_{2}(-n, \tau n+a, b ; \tau n+c,-n+d ; z)
$$

$\left(b \in \mathbb{C} \backslash \mathbb{Z}_{0}^{-} ; d \in \mathbb{C} \backslash \mathbb{N}_{0} ; \tau \neq 0 ; \tau n+a \neq 0,-1, \cdots,-n ; \tau n+c \neq 0,-1, \cdots,-n\right)$,

where

$$
\mathbb{Z}_{0}^{-}=\{0,-1,-2, \cdots\}=\mathbb{Z}^{-} \cup\{0\} .
$$

We also consider the asymptotic behavior of the zeros of the following Clausenian hypergeometric function:

$$
{ }_{3} F_{2}\left[\begin{array}{rr}
\left(k+\frac{1}{2}\right) n+l+\frac{1}{2},\left(k+\frac{1}{2}\right) n+l+1, k n+l ; & 1-z^{2} \\
(2 k+1) n+2 l+1, k n+l+1 ; &
\end{array}\right] .
$$

Numerical evidence and graphical illustrations of the clustering of the zeros on certain curves are generated by Mathematica (Version 4.0). 


\section{A SET OF MAIN RESUlts}

For notational simplicity, we write

$$
{ }_{3} F_{2}\left[\begin{array}{rr}
-n, \tau n+a, b ; \\
\tau n+c,-n+d ;
\end{array}\right]=: \sum_{k=0}^{n} a_{n, k} z^{k} \quad\left(a_{n, 0}:=1\right) .
$$

Lemma 1. Let

$b \in \mathbb{C} \backslash \mathbb{Z}_{0}^{-}, \quad d \in \mathbb{C} \backslash \mathbb{N}_{0}, \quad \tau \neq 0, \quad \tau n+a \neq 0,-1, \cdots,-n$ and $\tau n+c \neq 0,-1, \cdots,-n$.

Then each of the following inequalities holds true:

$$
\left|a_{n, k}\right| \leqq \mathfrak{M}_{0} \cdot(k+1)^{|d|+2|a-c|+|1-b|} \quad\left(0 \leqq k \leqq n ; n, k \in \mathbb{N}_{0}\right),
$$

where the constant $\mathfrak{M}_{0}>0$ depends on the parameters $a, b, c$ and $d$, but not on $n$ and $k$;

$$
\left|\frac{a_{n, n-k}}{a_{n, n}}\right| \leqq \widetilde{\mathfrak{M}}_{0} \cdot(k+2)^{|d|+2|c-a|+|1-b|} \quad\left(0 \leqq k \leqq n ; n, k \in \mathbb{N}_{0}\right),
$$

where the constant $\widetilde{\mathfrak{M}}_{0}>0$ depends on the parameters $a, b, c$ and $d$, but not on $n$ and $k$.

Proof. We first consider the case when $0<k<n \quad(n, k \in \mathbb{N})$. Then, according to the following identity for the Pochhammer symbol $(\lambda)_{n}$ defined by (1.2):

$$
(-n)_{j}=: \begin{cases}\frac{(-1)^{j} n !}{(n-j) !} & \left(0 \leqq j \leqq n ; n, j \in \mathbb{N}_{0}\right) \\ 0 & \left(j \geqq n+1 ; n \in \mathbb{N}_{0} ; j \in \mathbb{N}\right),\end{cases}
$$

we have

$$
\frac{a_{n, k+1}}{a_{n, k}}=\frac{(n-k)(\tau n+a+k)(b+k)}{(n-d-k)(\tau n+c+k)(k+1)} .
$$

We also observe that

$$
\begin{gathered}
\frac{n-k}{n-d-k}=1+\frac{d}{n-d-k}, \\
\frac{\tau n+a+k}{\tau n+c+k}=1+\frac{a-c}{\tau n+c+k}
\end{gathered}
$$

and

so that

$$
\frac{b+k}{k+1}=1+\frac{b-1}{k+1},
$$

$$
\begin{gathered}
\ln \left(\frac{n-k}{|n-d-k|}\right) \leqq \frac{|d|}{|n-d-k|}, \\
\ln \left(\frac{|\tau n+a+k|}{|\tau n+c+k|}\right) \leqq \frac{|a-c|}{|\tau n+c+k|}
\end{gathered}
$$

and

$$
\ln \left(\frac{|b+k|}{k+1}\right) \leqq \frac{|b-1|}{k+1},
$$


respectively. Hence, we have

$$
\begin{aligned}
\ln \left|a_{n, k}\right| & =\ln \left(\prod_{l=0}^{k-1} \frac{\left|a_{n, l+1}\right|}{\left|a_{n, l}\right|}\right)=\sum_{l=0}^{k-1} \ln \frac{\left|a_{n, l+1}\right|}{\left|a_{n, l}\right|} \\
& \leqq \sum_{l=0}^{k-1}\left(\frac{|d|}{|n-d-l|}+\frac{|a-c|}{|\tau n+c+l|}+\frac{|b-1|}{l+1}\right) \\
& \leqq \sum_{l=0}^{k-1}\left(\frac{|d|}{|n-d-k+l+1|}+\frac{2|a-c|}{l+1}+\frac{|b-1|}{l+1}\right) \quad(n \text { sufficiently large }) \\
& \leqq \sum_{l=0}^{[|d|]} \frac{|d|}{|n-d-k+1+l|}+\sum_{l=0}^{k-1} \frac{|d|+2|a-c|+|b-1|}{l+1} \\
& \leqq \mathfrak{M}+(|d|+2|a-c|+|b-1|)[\ln (k+1)+\gamma],
\end{aligned}
$$

where $\mathfrak{M}>0$ is a constant and $\gamma$ is the Euler-Mascheroni constant given by

$$
\gamma:=\lim _{n \rightarrow \infty}\left(\sum_{k=1}^{n} \frac{1}{k}-\ln n\right) \cong 0.577215664901532860606512 \cdots .
$$

It follows from (2.6) that

$$
\left|a_{n, k}\right| \leqq \mathfrak{M}_{0} \cdot(k+1)^{|d|+2|a-c|+|1-b|} \quad(0<k<n ; n, k \in \mathbb{N}),
$$

where the constant $\mathfrak{M}_{0}>0$ depends on the parameters $a, b, c$ and $d$, but not on $n$ and $k$. The cases of the assertion (2.2) of Lemma 1 when $k=0$ and $k=n$ are trivial.

Next, by replacing $k$ by $n-k$ in (2.5), we have

$$
\frac{a_{n, n-k}}{a_{n, n-k+1}}=\frac{[(\tau+1) n+c-k](k-d)(n-k+1)}{[(\tau+1) n+a-k] k(b+n-k)} .
$$

Since

$$
\frac{k-d}{k}=1-\frac{d}{k}, \quad \frac{(\tau+1) n+c-k}{(\tau+1) n+a-k}=1+\frac{c-a}{(\tau+1) n+a-k}
$$

and

it follows that

$$
\frac{n-k+1}{b+n-k}=1+\frac{1-b}{b+n-k}
$$

$$
\begin{gathered}
\ln \left(\frac{|k-d|}{k}\right) \leqq \frac{|d|}{k}, \\
\ln \left(\frac{|(\tau+1) n+c-k|}{|(\tau+1) n+a-k|}\right) \leqq \frac{|c-a|}{|(\tau+1) n+a-k|}
\end{gathered}
$$

and

$$
\ln \left(\frac{1+n-k}{|b+n-k|}\right) \leqq \frac{|1-b|}{|b+n-k|},
$$


respectively. Therefore, we obtain

$$
\begin{aligned}
\ln \left(\frac{\left|a_{n, n-k}\right|}{\left|a_{n, n}\right|}\right) & =\ln \left(\prod_{l=1}^{k} \frac{\left|a_{n, n-l}\right|}{\left|a_{n, n-l+1}\right|}\right)=\sum_{l=1}^{k} \ln \frac{\left|a_{n, n-l}\right|}{\left|a_{n, n-l+1}\right|} \\
& \leqq \sum_{l=1}^{k}\left(\frac{|d|}{l}+\frac{|c-a|}{|(\tau+1) n+a-l|}+\frac{|1-b|}{|b+n-l|}\right) \\
& \leqq \sum_{l=1}^{k}\left(\frac{|d|+2|c-a|}{l}+\frac{|1-b|}{|b+n-k-1+l|}\right) \text { (n sufficiently large) } \\
& \leqq \sum_{l=1}^{[|b|]+1} \frac{|1-b|}{|b+n-k-1+l|}+\sum_{l=1}^{k} \frac{|d|+2|c-a|+|1-b|}{l} \\
& \leqq \mathfrak{N}+(|d|+2|c-a|+|1-b|)[\ln (k+2)+\gamma],
\end{aligned}
$$

where $\mathfrak{N}>0$ is a constant and $\gamma$ is the Euler-Mascheroni constant given by (2.7). Consequently, it follows from (2.8) that

$$
\frac{\left|a_{n, n-k}\right|}{\left|a_{n, n}\right|} \leqq \widetilde{\mathfrak{M}}_{0} \cdot(k+2)^{|d|+2|c-a|+|1-b|},
$$

where the constant $\widetilde{\mathfrak{M}}_{0}>0$ depends on the parameters $a, b, c$ and $d$, but not on $n$ and $k$.

Finally, since the cases of the assertion (2.3) of Lemma 1 when $k=0$ and $k=n$ are immediate, our proof of Lemma 1 is completed.

Theorem 1. For fixed parameters $a, b, c, d$ and $\tau$ constrained by

$b \in \mathbb{C} \backslash \mathbb{Z}_{0}^{-}, \quad d \in \mathbb{C} \backslash \mathbb{N}_{0}, \quad \tau \neq 0, \quad \tau n+a \neq 0,-1, \cdots,-n$ and $\tau n+c \neq 0,-1, \cdots,-n$, the zeros of the Clausenian hypergeometric polynomials:

$$
{ }_{3} F_{2}\left[\begin{array}{rr}
-n, \tau n+a, b ; & z \\
\tau n+c,-n+d ;
\end{array}\right]
$$

approach the unit circle as $n \rightarrow \infty$.

Proof. From the assertion (2.2) of Lemma 1, it immediately follows that the following sequence of polynomials:

$$
\sum_{k=0}^{n} a_{n, k} z^{k}
$$

is uniformly bounded on the disk $\Omega$ given by

$$
\Omega=\{z: z \in \mathbb{C} \quad \text { and } \quad|z|<\rho \quad(0<\rho<1)\} .
$$

Furthermore, since (for fixed $k$ )

$$
a_{n, k}=\frac{(-n)_{k}(\tau n+a)_{k}(b)_{k}}{(\tau n+c)_{k}(-n+d)_{k}(1)_{k}} \rightarrow \frac{(b)_{k}}{(1)_{k}} \quad(n \rightarrow \infty),
$$

the following sequence of functions:

$$
\left\{\sum_{k=0}^{n} a_{n, k} z^{k}\right\}_{n \in \mathbb{N}_{0}}
$$


converges pointwise and, therefore, also uniformly, by Vitali's theorem [12, p. 252], to

$$
\sum_{k=0}^{\infty} \frac{(b)_{k}}{(1)_{k}} z^{k}={ }_{2} F_{1}\left[\begin{array}{c}
b, 1 ; \\
1 ;
\end{array}\right]=(1-z)^{-b} .
$$

Since the function $(1-z)^{-b}$ does not have any zeros inside the unit disk, by Hurwitz's theorem [12, p. 205], there exists an index $N_{0}$ such that

$$
\sum_{k=0}^{n} a_{n, k} z^{k}
$$

does not have zeros on $\Omega$ for $n>N_{0}$. Hence there exist numbers $\rho_{n}$ constrained by

$$
0<\rho_{n}<1 \text { so that } \rho_{n} \rightarrow 1
$$

and we can ensure that

$$
\rho_{n} \geqq \rho \quad\left(n>N_{0} ; 0<\rho<1\right) .
$$

Next, from the assertion (2.3) of Lemma 1, it also follows that the sequence of polynomials:

$$
\frac{z^{n}}{a_{n, n}} \sum_{k=0}^{n} a_{n, k}\left(\frac{1}{z}\right)^{k}=\sum_{k=0}^{n} \frac{a_{n, n-k}}{a_{n, n}} z^{k}
$$

is uniformly bounded on $\Omega$. Moreover, since (for fixed $k$ )

$$
\begin{aligned}
\frac{a_{n, n-k}}{a_{n, n}}=\frac{(-1)^{k} \Gamma(d) \Gamma(n+1) \Gamma[(\tau+1) n+a-k] \Gamma(n+b-k) \Gamma(2 n+c)}{k ! \Gamma(d-k) \Gamma(n+b) \Gamma[(\tau+1) n+c-k] \Gamma(n-k+1) \Gamma(2 n+a)} & \\
\rightarrow \frac{(1-d)_{k}}{(1)_{k}} \quad(n \rightarrow \infty), &
\end{aligned}
$$

the following sequence of functions:

$$
\left\{\sum_{k=0}^{n} \frac{a_{n, n-k}}{a_{n, n}} z^{k}\right\}_{n \in \mathbb{N}_{0}}
$$

converges pointwise, and therefore uniformly, by Vitali's theorem [12, p. 252], to

$$
\sum_{k=0}^{\infty} \frac{(1-d)_{k}}{(1)_{k}} z^{k}={ }_{2} F_{1}\left[\begin{array}{r}
1-d, 1 ; \\
1 ;
\end{array}\right]=(1-z)^{d-1} .
$$

Since the function $(1-z)^{d-1}$ does not have any zeros inside the unit disk, by Hurwitz's theorem [12, p. 205], there exists an index $N_{0}$ such that

$$
\frac{z^{n}}{a_{n, n}} \sum_{k=0}^{n} a_{n, k}\left(\frac{1}{z}\right)^{k}
$$

does not have zeros on $\Omega$ for $n>N_{0}$; that is,

$$
\sum_{k=0}^{n} a_{n, k} z^{k}
$$

does not have zeros $|z|>\frac{1}{\rho}$. Hence there exist numbers $\gamma_{n}$ constrained by

$$
\gamma_{n}>1 \text { so that } \gamma_{n} \rightarrow 1,
$$


and we can ensure that

$$
\gamma_{n} \leqq \frac{1}{\rho} \quad\left(n>N_{0} ; 0<\rho<1\right)
$$

Consequently, all zeros of the polynomial:

$$
\sum_{k=0}^{n} a_{n, k} z^{k}
$$

lie in the annulus given by

$$
\left\{z: z \in \mathbb{C} \quad \text { and } \quad \rho_{n} \leqq|z| \leqq \gamma_{n}\right\},
$$

which completes the proof of Theorem 1.

Upon setting

$$
a=1, \quad b=\frac{1}{2}, c=t+1, d=1-t \quad \text { and } \quad \tau=1
$$

in Theorem 1, we can deduce the following corollary.

Corollary 1 ([6, Theorem 2.2]). For fixed $t>0$, the zeros of the following Clausenian hypergeometric polynomials:

$$
{ }_{3} F_{2}\left[\begin{array}{r}
-n, n+1, \frac{1}{2} ; \\
n+t+1,-n+1-t ;
\end{array}\right]
$$

approach the unit circle as $n \rightarrow \infty$.

If, on the other hand, we choose

$$
a=-t, \quad b=t, \quad c=0, d=\frac{1}{2} \quad \text { and } \quad \tau=-2
$$

in Theorem 1, we obtain the following result.

Corollary 2. For fixed $t>0$, the zeros of the following Clausenian hypergeometric polynomials:

$$
{ }_{3} F_{2}\left[\begin{array}{c}
-n,-2 n-t, t ; \\
-2 n,-n+\frac{1}{2} ;
\end{array}\right]
$$

approach the unit circle $|z|=1$ as $n \rightarrow \infty$.

Remark 1. Corollary 2 provides an alternative form of a known result [10. Proposition 4.4].

Theorem 2. Let

$$
\tau n+a \neq 0,-1, \cdots,-n \text { and } \tau n+c \neq 0,-1, \cdots,-n .
$$

If the fixed parameters $a, b, c, d$ and $\tau$ are constrained by

$$
a \leqq c, \quad 0<b \leqq 1, d \leqq 0 \quad \text { and } \quad \tau>0
$$

or by

$$
a \geqq c, \quad 0<b \leqq 1, \quad d \leqq 0 \text { and } \tau<-1,
$$

then the zeros of the following Clausenian hypergeometric polynomials:

$$
{ }_{3} F_{2}\left[\begin{array}{cc}
-n, \tau n+a, b ; & z \\
\tau n+c,-n+d ;
\end{array}\right]
$$


lie outside the unit disk $|z| \leqq 1$ and approach the unit circle $|z|=1$ as $n \rightarrow \infty$.

The proof is based on the following classical theorem [14, p. 136].

Lemma 2 (Eneström-Kakeya Theorem [14, p. 136]). If

$$
0<a_{0}<a_{1}<\cdots<a_{n},
$$

then all zeros of the polynomial:

$$
p(z)=a_{0}+a_{1} z+\cdots+a_{n} z^{n}
$$

lie in the unit disk $|z| \leqq 1$.

Proof of Theorem 2. According to Theorem 1, we only need to prove that the zeros of the Clausenian hypergeometric polynomials:

$$
{ }_{3} F_{2}(-n, n+a, b ; n+c,-n+d ; z)
$$

lie outside the unit circle $|z|=1$. We also find from (2.1) that

$$
z_{3}^{n} F_{2}\left[\begin{array}{rr}
-n, \tau n+a, b ; & \frac{1}{z} \\
\tau n+c,-n+d ; & {[}
\end{array}\right]=\sum_{k=0}^{n} a_{n, k} z^{n-k}=\sum_{k=0}^{n} a_{n, n-k} z^{k} .
$$

Thus, under the parametric constraints:

$$
a \leqq c, 0<b \leqq 1, d \leqq 0 \text { and } \tau>0
$$

or

$$
a \geqq c, \quad 0<b \leqq 1, \quad d \leqq 0 \text { and } \tau<-1,
$$

which are already mentioned in the hypothesis of Theorem 2, we find from (2.5) (for sufficiently large $n$ ) that

$\frac{a_{n, n-(k+1)}}{a_{n, n-k}}=\frac{(k+1-d)[(\tau+1) n+c-k-1](n-k)}{(k+1)[(\tau+1) n+a-k-1](b+n-k-1)}>1 \quad(k=0,1, \cdots, n-1)$, which implies that the coefficients of the polynomial:

$$
\widetilde{F}(z):=z^{n}{ }_{3} F_{2}\left(-n, \tau n+a, b ; \tau n+c,-n+d ; z^{-1}\right)=\sum_{k=0}^{n} a_{n, n-k} z^{k}
$$

are positive and increasing:

$$
0<a_{n, n}<a_{n, n-1}<\cdots<a_{n, 0} .
$$

It follows from Lemma 2 that the zeros of the polynomial $\widetilde{F}(z)$ defined by $(2.11)$ lie in the unit disk $|z| \leqq 1$. Hence the zeros of

$$
{ }_{3} F_{2}(-n, \tau n+a, b ; \tau n+c,-n+d ; z)
$$

lie outside the unit disk $|z| \leqq 1$. This completes our proof of Theorem 2 .

Theorem 3. Let

$$
\tau n+a \neq 0,-1, \cdots,-n \text { and } \quad \tau n+c \neq 0,-1,-2, \cdots,-n .
$$

If the fixed parameters $a, b, c, d$ and $\tau$ are constrained by

$$
a \geqq c, \quad b \geqq 1, \quad 0 \leqq d<1 \quad \text { and } \quad \tau>0
$$

or by

$$
a \leqq c, \quad b \geqq 1, \quad 0 \leqq d<1 \quad \text { and } \quad \tau<-1,
$$


then the zeros of the following Clausenian hypergeometric polynomials:

$$
{ }_{3} F_{2}\left[\begin{array}{rr}
-n, \tau n+a, b ; & z \\
\tau n+c,-n+d ; &
\end{array}\right]
$$

lie inside the unit disk $|z| \leqq 1$ and approach the unit circle as $n \rightarrow \infty$.

Proof. By appealing appropriately to Theorem 1, we only need to prove that the zeros of

$$
{ }_{3} F_{2}(-n, n+a, b ; n+c,-n+d ; z)
$$

lie inside the unit disk $|z| \leqq 1$. Thus, under the parametric constraints:

$$
a \geqq c, \quad b \geqq 1, \quad 0 \leqq d<1 \text { and } \tau>0
$$

or

$$
a \leqq c, \quad b \geqq 1,0 \leqq d<1 \text { and } \tau<-1,
$$

which are already mentioned in the hypothesis of Theorem 2, we find from (2.5) (for sufficiently large $n$ ) that

$$
\frac{a_{n, k+1}}{a_{n, k}}=\frac{(n-k)(\tau n+a+k)(b+k)}{(n-d-k)(\tau n+c+k)(1+k)}>1 \quad(k=0,1, \cdots, n-1),
$$

which implies that the coefficients of

$$
{ }_{3} F_{2}(-n, \tau n+a, b ; \tau n+c,-n+d, z)
$$

are positive and increasing:

$$
0<a_{n, 0}<a_{n, 1}<\cdots<a_{n, n} .
$$

According to Lemma 2, we can see that the zeros of

$$
{ }_{3} F_{2}(-n, \tau n+a, b ; \tau n+c,-n+d, z)
$$

lie inside the unit disk $|z| \leqq 1$. This completes the proof of Theorem 3 .

We shall next use the following lemma, which we state here for the convenience of the interested reader.

Lemma 3 ([2, Theorem 2]). For arbitrary real numbers $k>0$ and $l \geqq 0$, the zeros of the following Gauss hypergeometric polynomials:

$$
{ }_{2} F_{1}\left[\begin{array}{rr}
-n, k n+l+1 ; & \\
k n+l+2 ; & z
\end{array}\right]
$$

cluster on the loop of the lemniscate given by

$$
\left|z^{k}(z-1)\right|=\frac{k^{k}}{(k+1)^{k+1}} \quad\left(\mathfrak{R}(z)>\frac{k}{k+1}\right)
$$

as $n \rightarrow \infty$.

Theorem 4. For arbitrary real numbers $k>0$ and $l \geqq 0$, the zeros of the following Clausenian hypergeometric function:

$$
{ }_{3} F_{2}\left[\begin{array}{rr}
\left(k+\frac{1}{2}\right) n+l+\frac{1}{2},\left(k+\frac{1}{2}\right) n+l+1, k n+l ; & 1-z^{2} \\
(2 k+1) n+2 l+1, k n+l+1 ; &
\end{array}\right]
$$


cluster on the loop given by

$$
\frac{|z-1|^{k}}{|z+1|^{k+1}}=\frac{k^{k}}{2(k+1)^{k+1}} \quad\left(\mathfrak{R}\left(\frac{z-1}{z+1}\right)>\frac{k}{k+1}\right)
$$

as $n \rightarrow \infty$.

Proof. By setting

$$
a=\left(k+\frac{1}{2}\right) n+l+\frac{1}{2}, \quad b=k n+l \quad \text { and } \quad \mu=1-z^{2}
$$

in the following hypergeometric reduction formula [16, p. 498, Equation (12)]:

$$
\begin{aligned}
& { }_{3} F_{2}\left[\begin{array}{rr}
a, a+\frac{1}{2}, b ; & \mu \\
2 a, b+1 ;
\end{array}\right] \\
& =\left[\frac{2}{\mu}(1-\sqrt{1-\mu})\right]^{2 b}{ }_{2} F_{1}\left[\begin{array}{rr}
b, 1+2 b-2 a ; & 1-\frac{2}{\mu}(1-\sqrt{1-\mu}) \\
b+1 ; &
\end{array}\right],
\end{aligned}
$$

we have

$$
\begin{aligned}
& { }_{3} F_{2}\left[\begin{array}{rr}
\left(k+\frac{1}{2}\right) n+l+\frac{1}{2},\left(k+\frac{1}{2}\right) n+l+1, k n+l ; & \\
(2 k+1) n+2 l+1, k n+l+1 ; &
\end{array}\right] \\
& =\left(\frac{2}{1+z}\right)^{2 n k+2 l}{ }_{2} F_{1}\left[\begin{array}{rr}
-n, k n+l+1 ; & z-1 \\
k n+l+2 ; & z+1
\end{array}\right] .
\end{aligned}
$$

We know from Lemma 3 that the zeros of the Gauss hypergeometric polynomials:

$$
{ }_{2} F_{1}\left[\begin{array}{rr}
-n, k n+l+1 ; & \frac{z-1}{z+1} \\
k n+l+2 ; &
\end{array}\right]
$$

will cluster on the curve given by

$$
\frac{|z-1|^{k}}{|z+1|^{k+1}}=\frac{k^{k}}{2(k+1)^{k+1}} \quad\left(\Re\left(\frac{z-1}{z+1}\right)>\frac{k}{k+1}\right)
$$

as $n \rightarrow \infty$. This completes our proof of Theorem 4 .

\section{NUMERICAL EVIDENCE AND GRAPHICAL ILLUSTRATIONS}

In this section, we make use of Mathematica (Version 4.0) in order to present numerical evidence and graphical illustrations for each of our results proved in the preceding section.

Figure 1 illustrates Theorem 1. It shows the location of the zeros of

$$
{ }_{3} F_{2}(-n, \tau n+a, b ; \tau n+c,-n+d ; z)
$$

for

$$
a=\frac{1}{2}, \quad b=\frac{1}{2}, \quad c=1, \quad d=\frac{3}{4} \quad \text { and } \quad \tau=1
$$

and various values of $n$, together with their asymptotic curve $|z|=1$. 
Figures 2 and 3 illustrate Theorem 2 by showing the zeros of the following Clausenian hypergeometric polynomials:

$$
{ }_{3} F_{2}\left(-n, \frac{3}{2} n+\frac{1}{3}, \frac{1}{4} ; \frac{3}{2} n+\frac{2}{3},-n-\frac{1}{2} ; z\right)
$$

and

$$
{ }_{3} F_{2}\left(-n,-\frac{3}{2} n+\frac{3}{4}, \frac{1}{2} ;-\frac{3}{2} n+\frac{1}{2},-n-\frac{1}{3} ; z\right),
$$

respectively, for various values of $n$, together with the unit circle (where they cluster as $n \rightarrow \infty)$.

Figures 4 and 5 illustrate Theorem 3 by showing the zeros of the following Clausenian hypergeometric polynomials:

$$
{ }_{3} F_{2}\left(-n, \frac{1}{2} n+\frac{2}{3}, \frac{3}{2} ; \frac{1}{2} n+\frac{1}{3},-n+\frac{1}{2} ; z\right)
$$

and

$$
{ }_{3} F_{2}\left(-n,-\frac{3}{2} n+\frac{1}{3}, \frac{3}{2} ;-\frac{3}{2} n+\frac{3}{4},-n+\frac{1}{2} ; z\right),
$$

respectively, for various values of $n$, together with their asymptotic curve $|z|=1$.

Figure 6 displays the zeros of the following Clausenian hypergeometric polynomials:

$$
\begin{array}{r}
{ }_{3} F_{2}\left[\left(k+\frac{1}{2}\right) n+l+\frac{1}{2},\left(k+\frac{1}{2}\right) n+l+1, k n+l ;\right. \\
\left.\quad(2 k+1) n+2 l+1, k n+l+1 ; 1-z^{2}\right]
\end{array}
$$

and the curve given by

$$
2(k+1)^{k+1}|z-1|^{k}=k^{k}|z+1|^{k+1} \quad \text { for } \quad k=\frac{1}{2}
$$

and various values of $n$ and $l$. It provides an illustration of Theorem 4 .
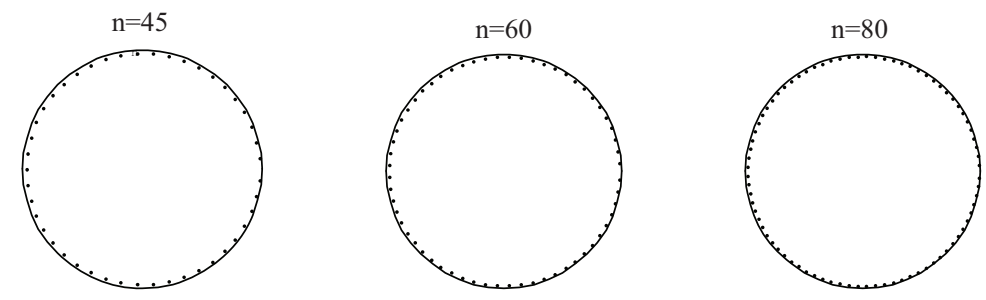

Figure 1. Zeros of ${ }_{3} F_{2}\left(-n, n+\frac{1}{2}, \frac{1}{2} ; n+1,-n+\frac{3}{4} ; z\right)$ and the unit disk. 

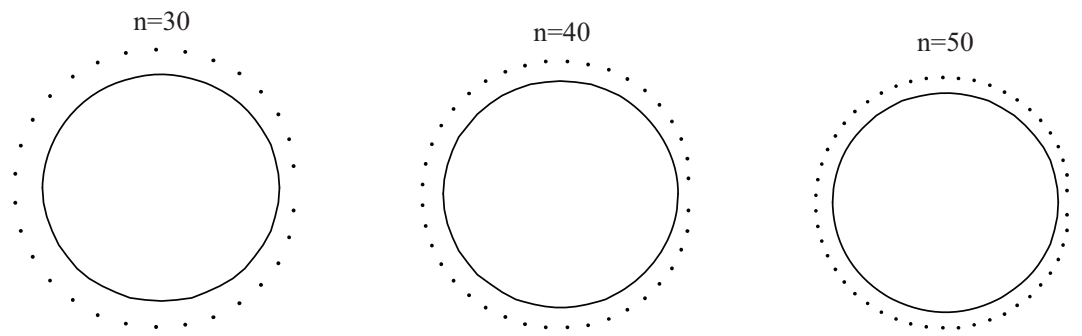

Figure 2. Zeros of ${ }_{3} F_{2}\left(-n, \frac{3}{2} n+\frac{1}{3}, \frac{1}{4} ; \frac{3}{2} n+\frac{2}{3},-n-\frac{1}{2} ; z\right)$ and the unit disk.
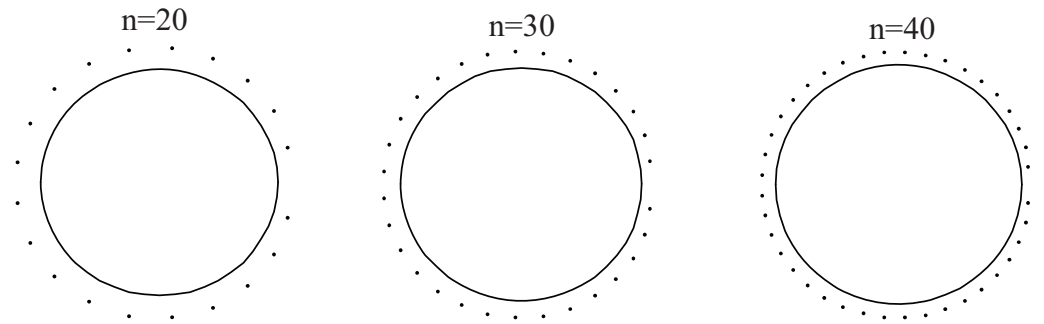

Figure 3. Zeros of ${ }_{3} F_{2}\left(-n,-\frac{3}{2} n+\frac{3}{4}, \frac{1}{2} ;-\frac{3}{2} n+\frac{1}{2},-n-\frac{1}{3} ; z\right)$ and the unit disk.


Figure 4. Zeros of ${ }_{3} F_{2}\left(-n, \frac{1}{2} n+\frac{2}{3}, \frac{3}{2} ; \frac{1}{2} n+\frac{1}{3},-n+\frac{1}{2} ; z\right)$ and the unit disk.
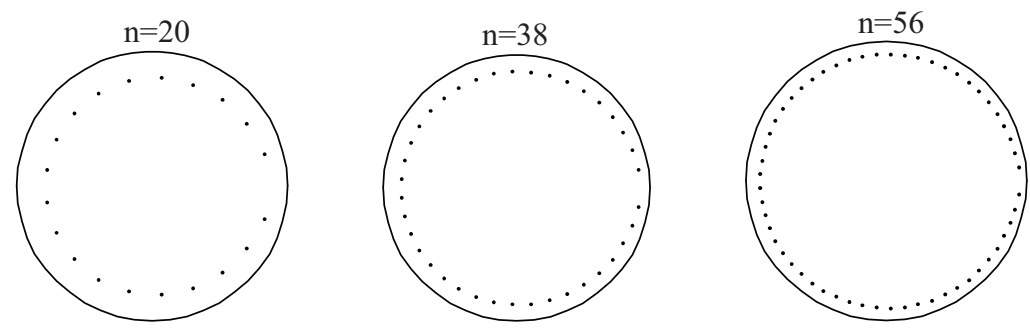

Figure 5. Zeros of ${ }_{3} F_{2}\left(-n,-\frac{3}{2} n+\frac{1}{3}, \frac{3}{2} ;-\frac{3}{2} n+\frac{3}{4},-n+\frac{1}{2} ; z\right)$ and the unit disk. 

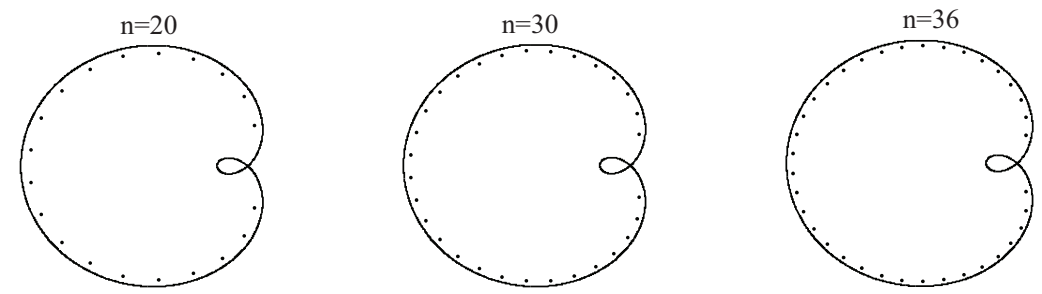

Figure 6. Zeros of ${ }_{3} F_{2}\left[\left(k+\frac{1}{2}\right) n+l+\frac{1}{2},\left(k+\frac{1}{2}\right) n+l+1, k n+l\right.$; $\left.(2 k+1) n+2 l+1, k n+l+1 ; 1-z^{2}\right]$ for $k=\frac{1}{2}$ and the curve $|z+1|^{3}=27|z-1|$.

\section{FURTHER REMARKS AND OBSERVATIONS}

In our present investigation, we have considered several interesting problems concerning the asymptotic distribution of the zeros of various classes of the Clausenian hypergeometric ${ }_{3} F_{2}$ functions and polynomials. In particular, we have made use of some classical analytic methods and techniques with a view to analyzing the behavior of the zeros of the Clausenian hypergeometric polynomials:

$$
{ }_{3} F_{2}(-n, \tau n+a, b ; \tau n+c,-n+d ; z),
$$

where $n$ is a nonnegative integer. We have also investigated some families of the hypergeometric ${ }_{3} F_{2}$ functions which are connected (by means of a hypergeometric reduction formula) with the Gauss hypergeometric polynomials of the form:

$$
{ }_{2} F_{1}(-n, k n+l+1 ; k n+l+2 ; z) .
$$

Furthermore, numerical evidence and graphical illustrations of the clustering of zeros on certain curves are generated by Mathematica (Version 4.0).

We conclude this paper by observing below some of the salient features of our investigation and some further comparisons of our results with those that are available in the existing literature on the subject.

Remark 2. In Theorem 1 (see also Figure 1), we have considered the problem involving the asymptotic distribution of the zeros of the following general Clausenian hypergeometric polynomials:

$$
\begin{array}{r}
{ }_{3} F_{2}\left[\begin{array}{c}
-n, \tau n+a, b ; \\
\tau n+c,-n+d ;
\end{array}\right] \\
\left(b \in \mathbb{C} \backslash \mathbb{Z}_{0}^{-} ; d \in \mathbb{C} \backslash \mathbb{N}_{0} ; \tau \neq 0 ; \tau n+a \neq 0,-1, \cdots,-n ; \tau n+c \neq 0,-1, \cdots,-n\right)
\end{array}
$$

when $n \rightarrow \infty$. Some known special cases and examples include (among others) the ${ }_{3} F_{2}$ polynomials:

$$
{ }_{3} F_{2}\left(-n, n+1, \frac{1}{2} ; n+t+1,-n-t+1 ; z\right),
$$

which were considered by Driver and Jordaan [6], and the ${ }_{3} F_{2}$ polynomials:

$$
{ }_{3} F_{2}\left(-n,-2 n-t, t ;-2 n,-n+\frac{1}{2} ; z\right),
$$

which were investigated by Driver and Möller [10]. 
Remark 3. In Theorem 2 (see also Figures 2 and 3) and in Theorem 3 (see also Figures 4 and 5), we have given two sufficient conditions so that the zeros approach the unit circle in different directions.

Remark 4. By applying Lemma 3 (see 2]), in Theorem 4 (see also Figure 6) we have obtained the asymptotic distribution of the zeros of the following Clausenian hypergeometric function:

$$
{ }_{3} F_{2}\left[\begin{array}{rr}
\left(k+\frac{1}{2}\right) n+l+\frac{1}{2},\left(k+\frac{1}{2}\right) n+l+1, k n+l ; & \\
(2 k+1) n+2 l+1, k n+l+1 ; &
\end{array}\right] \quad(k>0 ; l \geqq 0),
$$

which is capable of yielding several simpler results for different choices for the real numbers $k>0$ and $l \geqq 0$.

Remark 5. By appropriately specializing the various parameters in each of our main results (Theorems 1 to 4 above), one can deduce many other (known or new) potentially useful corollaries and consequences than those we have already indicated in the preceding sections.

\section{UNIFORM DISTRIBUTION OF THE ZEROS}

In our paper, the parameters $a, b, c, d$ and $\tau$ are independent of the large parameter $n$. Moreover, the zeros of the Clausenian hypergeometric polynomials:

$$
{ }_{3} F_{2}(-n, \tau n+a, b ; \tau n+c,-n+d ; z),
$$

are distributed uniformly along the curves (see Figures 1 to 6 ).

The zeros of the function $H_{n}(b, u)$, defined by

$$
\begin{aligned}
H_{n}(b, u) & :=u_{3}^{n} F_{2}\left(b,-b-2 n,-n ;-2 n,-n+\frac{1}{2} ; \frac{1}{u}\right) \\
& =u^{n}(1-z)^{b}\left[{ }_{2} F_{1}(-n, b ;-2 n ; z)\right]^{2}
\end{aligned}
$$

which were investigated in [10], are also distributed uniformly along the unit circle $|u|=1$ (see [10, Proposition 4.4]). However, the figures in 10] actually showed the distribution of the zeros of ${ }_{2} F_{1}(-n, b ;-2 n ; z)$, not the distribution of the zeros of the function $H_{n}(b, u)$ defined by $(5.1)$.

Since the transformation:

$$
(2 w-1)^{2}-1=-u
$$

in (5.1) would lead to two branches of $w$, the zeros of the Gauss hypergeometric polynomials:

$$
{ }_{2} F_{1}(-n, b ;-2 n ; z)
$$

are not distributed uniformly along the whole Cassini curve. In fact, the zeros of

$$
{ }_{2} F_{1}(-n, b ;-2 n ; z)
$$

are distributed only along the left branch of the curve (see [10]). 


\section{ACKNOWLEDGEMENTS}

The present investigation was supported by the Science Research Fund of Guangdong Provincial Education Department of the People's Republic of China under Grant LYM08101, the Science Research Fund of the Advanced Research Center of Foshan University and the Scientific Research Fund of Hunan Provincial Education Department of the People's Republic of China under Grant 08C118.

\section{REFERENCES}

[1] W. N. Bailey, Generalized Hypergeometric Series, Cambridge Tracts in Mathematics and Mathematical Physics, No. 32, Cambridge University Press, Cambridge, London and New York, 1935; Reprinted by Stechert-Hafner Service Agency, New York and London, 1964. MR0185155 (32:2625)

[2] K. Boggs and P. Duren, Zeros of hypergeometric functions, Comput. Methods Funct. Theory 1 (2001), 275-287. MR1931616 (2003k:33003)

[3] D. Dominici, K. Driver and K. Jordaan, Polynomial solutions of differential-difference equations, J. Approx. Theory (2009) [doi:10.1016/j.jat.2009.05.010].

[4] K. Driver and P. Duren, Zeros of the hypergeometric polynomials $F(-n, b ; 2 b ; z)$, Indag. Math. (New Ser.) 11 (2000), 43-51. MR.1809661(2002d:33006)

[5] K. Driver and K. Jordaan, Zeros of ${ }_{3} F_{2}(-n, b, c ; d, e ; z)$ polynomials, Numer. Algorithms 30 (2002), 323-333. MR1927508 (2003j:33018)

[6] K. Driver and K. Jordaan, Asymptotic zero distribution of ${ }_{3} F_{2}$ polynomials, Indag. Math. (New Ser.) 14 (2003), 319-327. MR2083078(2005f:33004)

[7] K. Driver and K. Jordaan, Separation theorems for the zeros of certain hypergeometric polynomials, J. Comput. Appl. Math. 199 (2007), 48-55. MR.2267530(2007m:33009)

[8] K. Driver and K. Jordaan, Pólya frequency sequences and real zeros of some ${ }_{3} F_{2}$ polynomials, J. Math. Anal. Appl. 332 (2007), 1045-1055. MR2324318 (2009m:33013)

[9] K. Driver, K. Jordaan and N. Mbuyi, Interlacing of zeros of linear combinations of classical orthogonal polynomials from different sequences, Appl. Numer. Math. 59 (2009), 2424-2429. MR2553144

[10] K. Driver and M. Möller, Zeros of the hypergeometric polynomials $F(-n, b ;-2 n ; z)$, J. Approx. Theory 110 (2001), 74-87. MR1826086 (2002c:33001)

[11] P. Duren and B. J. Guillou, Asymptotic Properties of zeros of hypergeometric polynomials, J. Approx. Theory 111 (2001), 329-343. MR.1849553 (2002f:33011)

[12] E. Hille, Analytic Function Theory, Vol. II, Chelsea Publishing Company, Bronx, New York, 1973. MR0201608 (34:1490)

[13] A. B. J. Kuijlaars, A. Martínez-Finkelshtein and R. Orive, Orthogonality of Jacobi polynomials with general parameters, Electron. Trans. Numer. Anal. 19 (2005), 1-17. MR 2149265 (2006e:33010)

[14] M. Marden, Geometry of Polynomials, American Mathematical Society, Providence, Rhode Island, 1996. MR0225972 (37:1562)

[15] P. Martínez-González and A. Zarzo, Higher order hypergeometric Lauricella function and zero asymptotics of orthogonal polynomials, J. Comput. Appl. Math. 233 (2010), 1577-1583.

[16] A. P. Prudnikov, Yu. A. Brychkov and O. I. Marichev, Integrals and Series, Vol. 3 (Moscow, Nauka, 1986 (in Russian); English translation, Gordon and Breach, New York, 1990); Errata in Math. Comput. 65 (1996), 1380-1384. MR.1054647 (91c:33001)

[17] H. M. Srivastava and P. W. Karlsson, Multiple Gaussian Hypergeometric Series, Halsted Press (Ellis Horwood Limited, Chichester), John Wiley and Sons, New York, Chichester, Brisbane and Toronto, 1985. MR834385 (87f:33015)

[18] H. M. Srivastava and H. L. Manocha, A Treatise on Generating Functions, Halsted Press (Ellis Horwood Limited, Chichester), John Wiley and Sons, New York, Chichester, Brisbane and Toronto, 1984. MR750112 (85m:33016)

[19] G. Szegö, Orthogonal Polynomials, Fourth edition, American Mathematical Society Colloquium Publications, Vol. 23, American Mathematical Society, Providence, Rhode Island, 1975. MR0372517 (51:8724) 
[20] N. M. Temme, Large parameter cases of the Gauss hypergeometric function, J. Comput. Appl. Math. 153 (2003), 441-462. MR.1985714(2004f:33006)

[21] J.-R. Zhou and Y.-Q. Zhao, An infinite asymptotic expansion for the extreme zeros of the Pollaczek polynomials, Stud. Appl. Math. 118 (2007), 255-279. MR2305779(2008e:33025)

Department of Mathematics and Statistics, University of Victoria, Victoria, British Columbia V8W 3R4, CANada

E-mail address: harimsri@math.uvic.ca

Department of Mathematics, Foshan University, Foshan 528000, Guangdong, People's RePublic of China

E-mail address: zhoujianrong2008@yahoo.com.cn

School of Mathematics and Computing Science, Changsha University of Science and Technology, Yuntang Campus, Changsha 410114, Hunan, People's Republic of China

E-mail address: wangmath@163.com 